\title{
Scanning tunneling spectroscopy of transition-metal-doped GaSb
}

\author{
P. Hidalgo, B. Méndez, and J. Piqueras \\ Departamento de Física de Materiales, Facultad de Físicas, Universidad Complutense, E-28040 Madrid, Spain \\ P. S. Dutta \\ Department of Electrical, Computer and Systems Engineering, Rensselaer Polytechnic Institute, Troy, New York 12180 \\ E. Dieguez \\ Departamento de Física de Materiales, Universidad Autónoma de Madrid, 28049 Madrid, Spain
}

(Received 12 March 1999; revised manuscript received 26 May 1999)

\begin{abstract}
V- and Ru-doped GaSb crystals have been investigated by scanning tunneling spectroscopy in a combined scanning electron microscope-scanning tunneling microscope system. Local variations of surface band gap have been measured with high spatial resolution. Precipitates in both kinds of doped samples show a nearly metallic behavior. The surface band gaps in the GaSb matrix have been found to depend on the dopant. [S0163-1829(99)06439-5]
\end{abstract}

\section{INTRODUCTION}

$\mathrm{GaSb}$ is emerging as an optoelectronic material to be used as substrate for various lattice matched devices operating in the infrared range (for a recent review, see Ref. 1). Doping of $\mathrm{GaSb}$ is a technological important subject that has been investigated in the past years by few groups. In particular, we have previously used cathodoluminescence (CL) to study ${ }^{2}$ the effect of transition metals $(\mathrm{Cr}, \mathrm{V}, \mathrm{Ru})$ on the native acceptor concentration. These dopants were found to reduce the acceptor concentration to a varying extent due to the different precipitation behavior. One effect of doping would be to shift the Fermi level towards the middle of the band gap. In the case of Ruthenium the reduction of acceptor concentration is more effective for low doping levels, which is attributed to the formation of precipitates and a lower incorporation in the lattice sites of the matrix for higher doping. For $\mathrm{Cr}$ and $\mathrm{V}$ doping the increase in impurity concentration causes a corresponding decrease in the native acceptor luminescence band. This is due to the formation of an impuritygallium vacancy center instead of the antisite related acceptor center ${ }^{2,3}$ present in undoped crystals. These results suggest that in the precipitate-free regions of highly $\mathrm{Ru}-$ doped samples the electronic behavior would be similar to that of the undoped material while in V-doped samples, with a high incorporation of dopant in the matrix, some differences could be observed. Such behavior has been in fact observed by CL microscopy in Ref. 2. Luminescence spectra of precipitate-free areas of high-doped GaSb:Ru crystals are similar to those of undoped samples and different from the $\mathrm{GaSb}: \mathrm{V}$ spectra that do not show the native acceptor band. The mentioned CL technique, used in a scanning electron microscope (SEM), has a space resolution in the micron or submicron range. On the other hand, scanning tunneling microscopy (STM) and spectroscopy enable us to investigate the electronic properties of semiconductors with higher resolution. In particular, current imaging tunneling spectroscopy ${ }^{4}$ (CITS) provides images that reveal nanometer scale variations in the surface electronic structure of the samples. ${ }^{5}$ In the present work STM and CITS have been applied to investigate the local surface electronic properties of $\mathrm{GaSb}$, $\mathrm{GaSb}: \mathrm{V}$, and GaSb:Ru crystals. The study has been performed in a SEM/STM combined instrument that enables us to carry out STM-CITS measurements in well-defined areas, as precipitates or precipitate-free regions.

\section{EXPERIMENTAL METHOD}

The samples used in this work were GaSb, GaSb:V, and $\mathrm{GaSb}: \mathrm{Ru}$ crystals grown by the vertical Bridgmann technique by tip nucleation without a seed. ${ }^{1}$ The doping of the crystals was done by adding the respective metals to the melt at the level of $10^{19}$ atoms $\mathrm{cm}^{-3}$. The crystals were $12 \mathrm{~mm}$ in diameter and about $40 \mathrm{~mm}$ in length. During growth a gradient of the dopant concentration arises along the crystals. In the dopant rich end of the ingots precipitates are readily observed in the SEM. ${ }^{2}$ Disks were cut perpendicular to the growth axis at a place close to the precipitate-rich end of the $\mathrm{V}$ - and Ru-doped ingots and the wafers were chemomechanically polished to a mirror finish. For the STM measurements the combined SEM-STM based on a Leica 440 SEM operating under a vacuum of $10^{-6}$ Torr was used. The small size of the STM enabled it to be mounted on the SEM specimen holder. Electrochemically etched or mechanically sharpened Pt-Ir and Au wires were used as STM probe tips. For CITS measurements the constant-current topograph was obtained in a $128 \times 128$ pixel grid, the feedback loop interrupted for 2 $\mathrm{ms}$, and the voltage digitally ramped from the tunnel voltage to a set of 44 predetermined values while the current was sampled. This provides 44 tunneling files at different voltages in addition to the current constant topograph. To analyze the data, the normalized differential conductance, $(d I / d V) /(I / V)$, spectra were used. In every pixel ten $I-V$ curves were recorded and averaged. This quantity provides a rather direct measure of the surface density of states. ${ }^{6}$ For the STM measurements the samples were cleaned with hydrochloric acid and acetone to remove oxides, dried in a flow of 


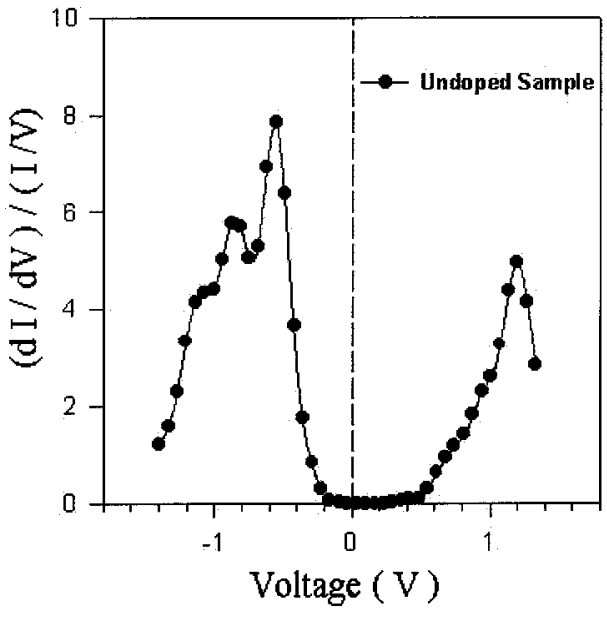

FIG. 1. Normalized differential conductance spectrum of an undoped sample.

dry nitrogen, and inmediately mounted in the microscope chamber.

\section{RESULTS AND DISCUSSION}

STM and CITS images were recorded in both undoped and doped samples. CITS images of all samples show contrast with high spatial resolution due to local conductance variations. The contrast appears for voltages below and above the bulk band gap and is higher in the doped samples. Further information on the local properties of the samples is obtained from the normalized differential conductance curves. The curves were plotted from the data of the CITS images corresponding to the 44 selected voltage values by averaging the data on regions of about $20 \mathrm{~nm} \times 20 \mathrm{~nm}$. The undoped GaSb crystals show almost featureless secondaryelectron SEM images while the corresponding CL images reveal the presence of subgrain boundaries in a bright homogeneous background. ${ }^{7}$ Figure 1 shows a typical normalized differential conductance curve of our undoped crystals in which a surface band gap of about $800 \mathrm{meV}$ is observed. This curve, as well as the other conductance curves, was represented from the average of the data obtained in a square region of about $10-\mathrm{nm}$ side. This is close to the bulk value of $795 \mathrm{meV}$ of $\mathrm{GaSb}$ measured in the CL spectra. ${ }^{7}$ The $p$-type behavior of as-grown undoped $\mathrm{GaSb}$ is revealed by the asymmetry of the curve relative to the zero-bias voltage, which corresponds to the Fermi level. These data and the reproducibility of the curves obtained with different tips and under different tunneling conditions show that the curves are representative of the $\mathrm{GaSb}$ although they were recorded in the SEM high vacuum. Also, the main features of the conductance curves of the doped samples, described below, were found to be reproducible. Figure 2 shows the secondary electron (SE) image of a vanadium-doped sample in which precipitates are observed. X-ray microanalysis reveals that the atomic percentage in the precipitates is $24.5 \% \mathrm{Ga}, 51.5 \% \mathrm{Sb}$, and $24 \% \mathrm{~V}$, while in the precipitate free regions the $\mathrm{V}$ atomic percentage is too low to be detected by this technique. The conductance curves of $\mathrm{GaSb}: \mathrm{V}$ have been found to be different from those of the undoped crystals and to depend on the region, precipitate or matrix, considered. This

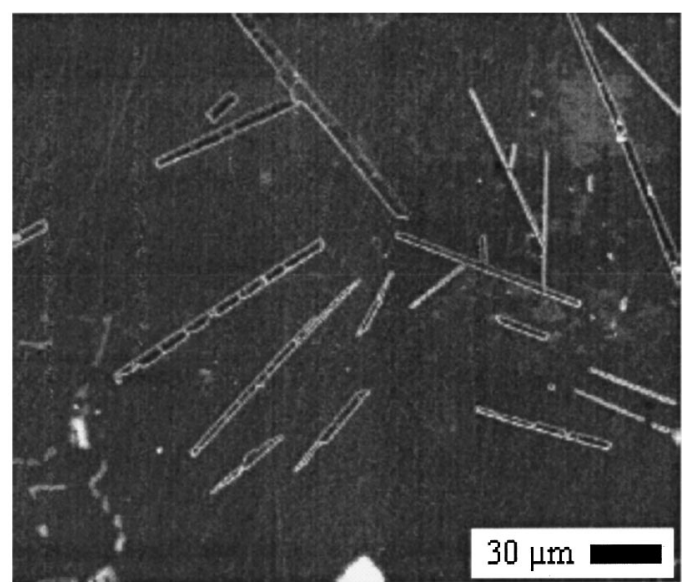

FIG. 2. Secondary electron image of a GaSb:V crystal showing precipitates.

effect is observed in the spectra of Figure 3 corresponding to a precipitate-free area, in which a surface gap of about 0.5 $\mathrm{eV}$ is measured, and to a precipitate showing a nearly metallic behavior. The dopant-induced shift of the Fermi level is revealed in the curve of the precipitate-free area by the centered position of the zero-bias voltage.

The GaSb:Ru crystals also contain precipitates that are observed in the SEM (Fig. 4). The atomic percentage in the precipitates measured by $\mathrm{x}$-ray microanalysis is $56.6 \% \mathrm{Ga}$, $33.4 \% \mathrm{Sb}$, and $10 \% \mathrm{Ru}$ while outside the precipitates ruthenium was not detected. The normalized differential conductance spectra recorded in a precipitate and in the matrix are shown in Fig. 5. It is observed that in the precipitate-free region the curves are similar to those of the undoped crystals. However the zero-bias voltage appears shifted towards the middle as in the $\mathrm{V}$-doped samples. In the precipitates the gap value is about $0.1-0.2 \mathrm{eV}$. The differences in the conductance curves of $\mathrm{V}$ - and Ru-doped crystals agree with the suggestion, stated in the Introduction, that in the case of Ruthenium there is a low incorporation in the lattice site

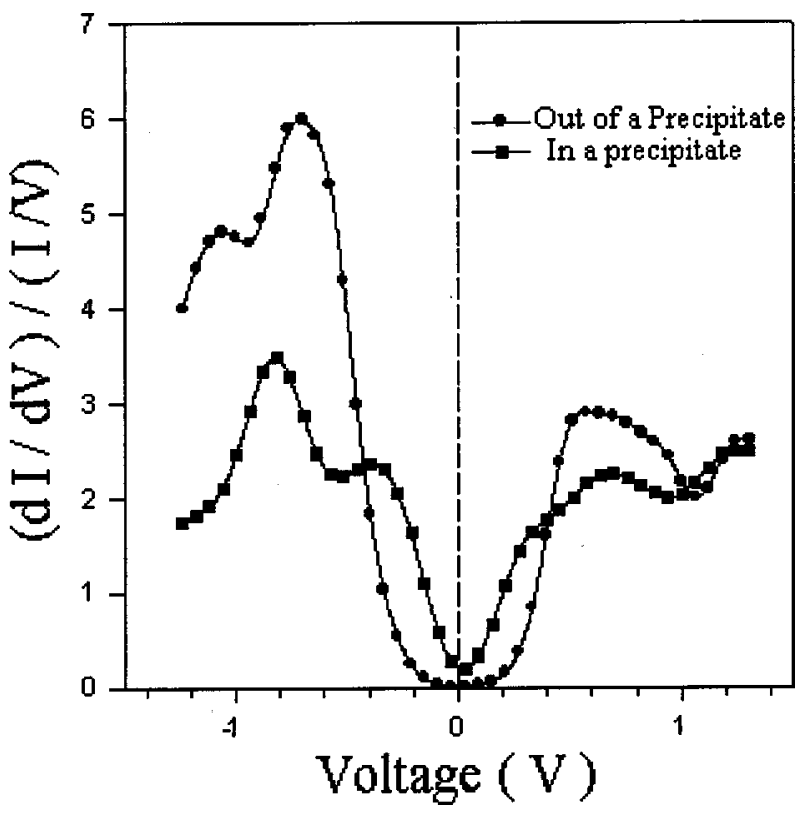

FIG. 3. Normalized differential conductance spectra of GaSb:V. 


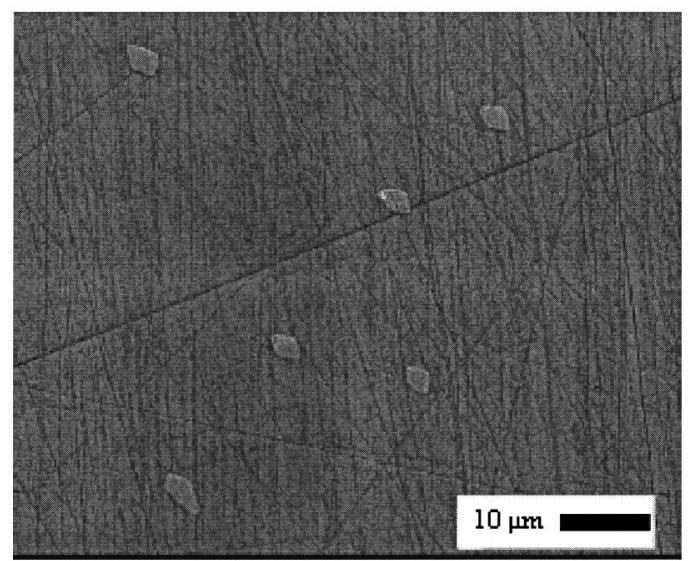

FIG. 4. Secondary electron image of precipitates in GaSb:Ru.

matrix for high doping levels. Consequently the conductance curves and, in particular, the surface band gap of $\mathrm{GaSb}: \mathrm{Ru}$ and of the undoped crystals are similar. On the contrary, highly doped GaSb:V crystals present not only precipitates containing vanadium but also an appreciable vanadium incorporation to the matrix as concluded by the reduction of native acceptors observed by CL. This would cause the different behavior, observed by tunneling spectroscopy in this work, of GaSb:V and GaSb:Ru crystals.

\section{SUMMARY}

In summary, the effect of high $\mathrm{V}$ and $\mathrm{Ru}$ concentrations on the electronic properties of GaSb has been studied with a

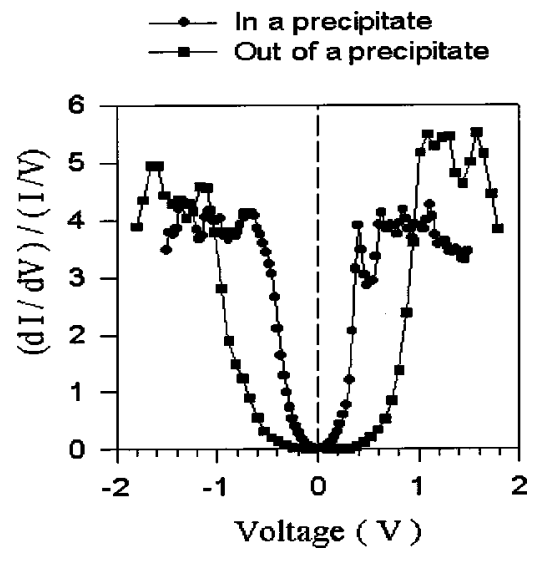

FIG. 5. Normalized differential conductance spectra recorded (a) in a precipitate and (b) in a precipitate-free area of GaSb:Ru.

SEM-STM instrument. STM-CITS reveals local variations in the surface band gap with high spatial resolution. The precipitates of both kinds of doped samples show a nearly metallic behavior. In the matrix region of the $\mathrm{V}$-doped crystals either a surface gap of $0.5 \mathrm{eV}$ or a metallic behavior is observed. The matrix of the Ru-doped samples present the same band gap as the undoped crystals, about $0.8 \mathrm{eV}$. The difference between $\mathrm{V}$ and $\mathrm{Ru}$ is explained by a higher incorporation to the matrix lattice sites in the case of vanadium atoms.

\section{ACKNOWLEDGMENTS}

This work was supported by DGES (PB96-0639) and by CICYT (ESP98-1340) (MAT98-1306E).
${ }^{1}$ P. S. Dutta, H. L. Baht, and V. Kumar, J. Appl. Phys. 81, 5821 (1997).

${ }^{2}$ P. Hidalgo, B. Méndez, P. S. Dutta, J. Piqueras, and E. Dieguez, Phys. Rev. B 57, 6479 (1998).

${ }^{3}$ E. I. Georgitsé, L. M. Gutsulyak, V. I. Ivanov-Omskil, V. A. Smirnov, and Sh. U. Yuldashev, Fiz. Tekh. Poluprovodn. 25, 1960 (1991) [Sov. Phys. Semicond. 25, 1180 (1991)].
${ }^{4}$ R. J. Hammers, R. M. Tromp, and J. E. Demuth, Phys. Rev. Lett. 56, 1972 (1986).

${ }^{5}$ R. M. Feenstra, Surf. Sci. 299/300, 965 (1994).

${ }^{6}$ R. M. Feenstra, J. A. Stroscio, and A. P. Fein, Surf. Sci. 181, 295 (1987).

${ }^{7}$ B. Méndez, J. Piqueras, P. S. Dutta, and E. Dieguez, Mater. Sci. Eng., B B42, 38 (1996). 